УДК 519.4

\author{
I. Protasov, K. Protasova
}

\title{
CLOSENESS AND LINKNESS IN BALLEANS
}

I. Protasov, K. Protasova. Closeness and linkness in balleans, Mat. Stud. 53 (2020), 100-108.

A set $X$ endowed with a coarse structure is called ballean or coarse space. For a ballean $(X, \mathcal{E})$, we say that two subsets $A, B$ of $X$ are close (linked) if there exists an entourage $E \in \mathcal{E}$ such that $A \subseteq E[B], B \subseteq E[A]$ (either $A, B$ are bounded or contain unbounded close subsets). We explore the following general question: which information about a ballean is contained and can be extracted from the relations of closeness and linkness.

1. Introduction. Given a set $X$, a family $\mathcal{E}$ of subsets of $X \times X$ is called a coarse structure on $X$ if

- each $E \in \mathcal{E}$ contains the diagonal $\triangle_{X}, \triangle_{X}=\{(x, x) \in X: x \in X\}$;

- if $E, E^{\prime} \in \mathcal{E}$ then $E \circ E^{\prime} \in \mathcal{E}$ and $E^{-1} \in \mathcal{E}$, where $E \circ E^{\prime}=\{(x, y): \exists z((x, z) \in E$, $\left.\left.(z, y) \in E^{\prime}\right)\right\}, E^{-1}=\{(y, x):(x, y) \in E\}$;

- if $E \in \mathcal{E}$ and $\triangle_{X} \subseteq E^{\prime} \subseteq E$ then $E^{\prime} \in \mathcal{E}$;

- $\bigcup \mathcal{E}=X \times X$.

A subfamily $\mathcal{E}^{\prime} \subseteq \mathcal{E}$ is called a base for $\mathcal{E}$ if, for every $E \in \mathcal{E}$, there exists $E^{\prime} \in \mathcal{E}^{\prime}$ such that $E \subseteq E^{\prime}$. For $x \in X, A \subseteq X$ and $E \in \mathcal{E}$, we denote $E[x]=\{y \in X:(x, y) \in E\}$, $E[A]=\bigcup_{a \in A} E[a]$ and say that $E[x]$ and $E[A]$ are balls of radius $E$ around $x$ and $A$.

The pair $(X, \mathcal{E})$ is called a coarse space [14] or a ballean [11], [13].

For a ballean $(X, \mathcal{E})$, a subset $B \subseteq X$ is called bounded if $B \subseteq E[x]$ for some $E \in \mathcal{E}$ and $x \in X$. A ballean $(X, \mathcal{E})$ is called bounded (unbounded) if $X$ is bounded (unbounded). The family $\mathcal{B}_{(X, \mathcal{E})}$ of all bounded subsets of $(X, \mathcal{E})$ is called the bornology of $(X, \mathcal{E})$. We denote

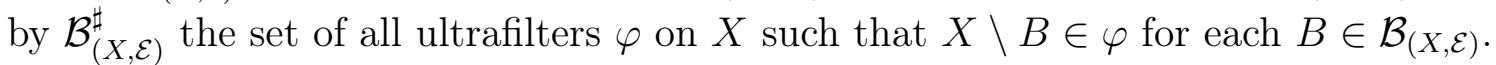

Definition 1. Given a ballean $(X, \mathcal{E})$, we say that subsets $A, B$ of $X$ are

- close (write $A \delta B$ ) if there exists $E \in \mathcal{E}$ such that $A \subseteq E[B], B \subseteq E[A]$;

- linked (write $A \lambda B$ ) if either $A, B$ are bounded or there exist unbounded subsets $A^{\prime} \subseteq A$, $B^{\prime} \subseteq B$ such that $A^{\prime} \delta B^{\prime}$.

The relations $\delta$ and $\lambda$, called closeness and linkness, are special kinds of asymptotic proximities [9]. We note that $\delta$ and $\lambda$ play the central part in ultrafilter description of the Higson's corona $\nu(X, \mathcal{E})$ of $(X, \mathcal{E})$, see [8] and Section 6. The negation of $\lambda$ (namely,

2020 Mathematics Subject Classification: 54E99, 54D80.

Keywords: coarse structure; ballean; coarse space; closeness; linkness; rigidity; stability. doi:10.30970/ms.53.1.100-108

(C) I. Protasov, K. Protasova, 2020 
asymptotic disjointness) is used for definition of normal balleans, see [7] and Section 4. The relation $\delta$ is an equivalence and each $\delta$-class is a connected component in the hyperballean of $(X, \mathcal{E})$, see [5], [12].

In above and other cases, $\delta$ and $\lambda$ were used as a tool for studing balleans, but $\delta$ and $\lambda$ are interesting for their own sake. In this paper, we concentrate around the following general questions:

Which properties of a ballean $(X, \mathcal{E})$ can be recognized by $\delta_{(X, \mathcal{E})}$ or $\lambda_{(X, \mathcal{E})}$ ?

How can one detect if a ballean $(X, \mathcal{E})$ is uniquely determined by $\lambda_{(X, \mathcal{E})}$ or $\delta_{(X, \mathcal{E})}$ ?

To make these questions more precise, we need some definitions.

Definition 2. We say that two balleans $(X, \mathcal{E}),\left(X, \mathcal{E}^{\prime}\right)$ are $\delta$-equivalent ( $\lambda$-equivalent) if $\delta_{(X, \mathcal{E})}=\delta_{\left(X, \mathcal{E}^{\prime}\right)}\left(\lambda_{(X, \mathcal{E})}=\lambda_{\left(X, \mathcal{E}^{\prime}\right)}\right)$.

Every $\delta$-equivalent balleans are $\lambda$-equivalent, but not vice versa, see Section 2 .

If $(X, \mathcal{E}),\left(X, \mathcal{E}^{\prime}\right)$, are $\lambda$-equivalent then $\mathcal{B}_{(X, \mathcal{E})}=\mathcal{B}_{\left(X, \mathcal{E}^{\prime}\right)}$.

Definition 3. A ballean $(X, \mathcal{E})$ is called $\delta$-rigid ( $\lambda$-rigid) if, for every coarse structure $\mathcal{E}^{\prime}$ on $X$ such that $\delta_{(X, \mathcal{E})}=\delta_{\left(X, \mathcal{E}^{\prime}\right)}\left(\lambda_{(X, \mathcal{E})}=\lambda_{\left(X, \mathcal{E}^{\prime}\right)}\right)$, we have $\mathcal{E}=\mathcal{E}^{\prime}$.

Thus, every $\delta$-rigid $\left(\lambda\right.$-rigid) ballean $(X, \mathcal{E})$ is uniquely determined by the relation $\delta_{(X, \mathcal{E})}\left(\lambda_{(X, \mathcal{E})}\right)$. Every $\lambda$-rigid ballean is $\delta$-rigid.

For balleans $(X, \mathcal{E})$, uniquely determined by $\mathcal{B}_{(\mathcal{X}, \mathcal{E})}$ see [4].

Definition 4. We say that a class $\mathcal{K}$ of balleans is

- $\delta$-stable $(\lambda$-stable $)$, for any two $\delta$-equivalent $\left(\lambda\right.$-equivalent) balleans $(X, \mathcal{E}),\left(X, \mathcal{E}^{\prime}\right)$, if $(X, \mathcal{E}) \in \mathcal{K}$ then $\left(X, \mathcal{E}^{\prime}\right) \in \mathcal{K}$

- $\delta$-rigid $(\lambda$-rigid $)$, for any two $\delta$-equivalent ( $\lambda$-equivalent) balleans $(X, \mathcal{E}),\left(X, \mathcal{E}^{\prime}\right) \in \mathcal{K}$, we have $\mathcal{E}=\mathcal{E}^{\prime}$.

By $[9$, Theorem 3.4], for every ballean $(X, \mathcal{E})$, there exists the strongest by inclusion coarse structure $\mathcal{E}_{\delta}$ on $X$ such that $(X, \mathcal{E}),\left(X, \mathcal{E}_{\delta}\right)$ are $\delta$-equivalent. Analogously, there exists the strongest by inclusion coarse structure $\mathcal{E}_{\lambda}$ on $X$ such that $(X, \mathcal{E}),\left(X, \mathcal{E}_{\lambda}\right)$ are $\lambda$-equivalent.

Definition 5. We say that a ballean $(X, \mathcal{E})$ is $\delta$-strong $(\lambda$-strong $)$ if $\mathcal{E}=\mathcal{E}_{\delta}\left(\mathcal{E}=\mathcal{E}_{\lambda}\right)$.

In Section 2 we prove that every discrete ballean is $\lambda$-rigid, every coarsely discrete ballean is $\delta$-rigid but needs not to be $\lambda$-rigid.

In Section 3 we show that the class of metrizable balleans is $\lambda$-rigid but not $\lambda$-stable, the class of submetrizable balleans is $\lambda$-stable. Every metrizable ballean $(X, \mathcal{E})$ is $\lambda$-strong (so $\delta$-strong) but $(X, \mathcal{E})$ is $\lambda$-rigid if and only if $(X, \mathcal{E})$ is discrete. Is every metrizable ballean $\delta$-rigid? This question remains open.

In Section 4 we prove that the class of normal balleans is $\lambda$-stable, but not $\delta$-rigid.

In Section 5 we prove that the class of finitary group balleans is $\lambda$-rigid.

In Section 6 we show that the parallelity relation on $\mathcal{B}_{(X, \mathcal{E})}^{\sharp}$ uniquely defines $\delta_{(X, \mathcal{E})}$ and apply this statement to balleans $(X, \mathcal{E})$ with finite $\mathcal{B}_{(X, \mathcal{E})}^{\sharp}$. We conclude the paper with an ultrafilter characterization of the normality.

2. Discrete balleans. We recall that a ballean $(X, \mathcal{E})$ is discrete if, for every $E \in \mathcal{E}$, there exists a bounded subset $B$ such that $E[x]=\{x\}$ for each $x \in X \backslash B$. A subset $Y \subseteq X$ is called discrete if the subballean $\left(Y,\left.\mathcal{E}\right|_{Y}\right)$ is discrete. 
Theorem 1. For a ballean $(X, \mathcal{E})$, the following statements are equivalent:

1. $(X, \mathcal{E})$ is discrete;

2. if $Y \subseteq X, Z \subseteq X$ and $Y \delta Z$ then $Y \backslash Z$ and $Z \backslash Y$ are bounded;

3. if $Y \subseteq X, Z \subseteq X$ and $Y \lambda Z$ then either $Y, Z$ are bounded or $Y \cap Z$ is unbounded.

Proof. The implications $(1) \Longrightarrow(2) \Longrightarrow(3)$ are evident.

We assume that $(3)$ holds but $(X, \mathcal{E})$ is not discrete. Then there exists $E \in \mathcal{E}$ such that the set $\{x \in X:|E[x]|>1\}$ is unbounded. We choose an unbounded subset $Y$ of $X$ such that $|E[y]|>1$ for each $y \in Y$ and $E[y] \cap E\left[y^{\prime}\right]=\varnothing$ for all distinct $y, y^{\prime} \in Y$. For each $y \in Y$, we pick $z_{y} \in E[y] \backslash\{y\}$ and denote $Z=\left\{z_{y}: y \in Y\right\}$. Clearly, $Y \lambda Z$ but $Y \cap Z=\varnothing$ contradicting (3).

Corollary 1. Every discrete ballean is $\lambda$-rigid and hence $\delta$-rigid.

To continue, we need the following construction from [6].

Let $(X, \mathcal{E})$ be an unbounded ballean and $\varphi$ be a filter on $X$ such that $X \backslash B \in \varphi$ for each $B \in \mathcal{B}_{(X, \mathcal{E})}$. We denote by $\mathcal{E}_{\varphi}$ a coarse structure on $X$ with the base $\left\{H_{(E, \phi)}: E \in \mathcal{E}, \Phi \in \varphi\right\}$, where

$$
H_{(E, \phi)}[x]= \begin{cases}\{x\} & \text { if } x \in \Phi, \\ E[x] \backslash \Phi & \text { if } x \in X \backslash \Phi .\end{cases}
$$

Clearly, $\mathcal{E}_{\varphi} \subseteq \mathcal{E}$ and $\mathcal{B}_{(X, \mathcal{E})}=\mathcal{B}_{\left(X, \mathcal{E}_{\varphi}\right)}$.

Theorem 2. Let $(X, \mathcal{E})$ be a ballean, $E_{0} \in \mathcal{E}$. Assume that there exists an unbounded subset $Y$ of $X$ such that $\left|E_{0}[y]\right|>1, y \in Y$ and every unbounded subset of $Y$ can be partitioned in two unbounded subsets. Then there exists an ultrafilter $\varphi$ on $X$ such that $\mathcal{E}_{\varphi} \subsetneq \mathcal{E}$ and $(X, \mathcal{E}),\left(X, \mathcal{E}_{\varphi}\right)$ are $\lambda$-equivalent, so $(X, \mathcal{E})$ is not $\lambda$-rigid.

Proof. We take an arbitrary ultrafilter $\varphi \in \mathcal{B}_{(X, \mathcal{E})}^{\sharp}$ such that $Y \in \varphi$. The definition of the coarse structure $\mathcal{E}_{\varphi}$ ensures that $\mathcal{E}_{\varphi} \subsetneq \mathcal{E}$.

We take arbitrary subsets $A, B$ of $X$ linked in $(X, \mathcal{E})$ and show that $A, B$ are linked in $\left(X, \mathcal{E}_{\varphi}\right)$. If $A, B$ are bounded in $(X, \mathcal{E})$ then $A, B$ are bounded in $\left(X, \mathcal{E}_{\varphi}\right)$, so we suppose that $A, B$ are unbounded in $(X, \mathcal{E})$. We choose unbounded subsets $A^{\prime} \subseteq A$ and $B^{\prime} \subseteq B$ which are close in $(X, \mathcal{E})$. If $A^{\prime} \cap Y$ and $B^{\prime} \cap Y$ are bounded then $A^{\prime}, B^{\prime}$ are close in $\left(X, \mathcal{E}_{\varphi}\right)$ by the construction of $\mathcal{E}_{\varphi}$. We suppose that $A^{\prime} \cap Y$ is unbounded and partition $A^{\prime} \cap Y$ in two unbounded subsets $C, D$. Since $\varphi$ is an ultrafilter, either $C \notin \varphi$ or $D \notin \varphi$. Hence, either $C$ or $D$ is linked to $B^{\prime}$ in $\left(X, \mathcal{E}_{\varphi}\right)$.

Let $(X, \mathcal{E})$ be a ballean. We recall that a subset $Y$ of $X$ is large if there exists $E \in \mathcal{E}$ such that $X=E[Y]$.

Clearly, $Y$ is large if and only if $Y \delta X$. We note that every unbounded subset of $X$ is linked with $X$.

A ballean $(X, \mathcal{E})$ is called coarsely discrete if $X$ contains a large discrete subset.

Theorem 3. Every coarsely discrete ballean $(X, \mathcal{E})$ is $\delta$-rigid.

Proof. Assuming that a coarse structure $\mathcal{E}^{\prime}$ on $X$ is $\delta$-equivalent to $\mathcal{E}$, we shall prove that $\mathcal{E}^{\prime}=\mathcal{E}$. 
Let $Y$ be a large discrete subset of $(X, \mathcal{E})$. Then there exists $E \in \mathcal{E}$ such that $E[Y]=X$. Since $Y \delta X$, there exists $E^{\prime} \in \mathcal{E}^{\prime}$ such that $E^{\prime}[Y]=X$. The $\delta$-equivalence of the coarse structures $\mathcal{E}, \mathcal{E}^{\prime}$ implies the $\delta$-equivalence of the induced coarse structures $\left.\mathcal{E}\right|_{Y}$ and $\mathcal{E}_{Y}^{\prime}$. Now Corollary 1 implies that $\left.\mathcal{E}\right|_{Y}=\left.\mathcal{E}^{\prime}\right|_{Y}$. Taking into account that $Y$ is large in both balleans $(X, \mathcal{E})$ and $\left(X, \mathcal{E}^{\prime}\right)$, we conclude that $\mathcal{E}=\mathcal{E}^{\prime}$.

Example 1. We define a coarse structure $\mathcal{E}$ on the set $\mathbb{N}$ of natural numbers such that the ballean $(\mathbb{N}, \mathcal{E})$ is $\delta$-rigid but not $\lambda$-rigid. We denote

$$
A=\{(2 n+1,2 n+2),(2 n+2,2 n+1),(n, n): n \in \omega\}
$$

and put $\mathcal{E}=\{(F \times F) \cup A: F \subset \mathbb{N},|F|<\omega\}$. Since the set $2 \mathbb{N}$ is large and discrete in $(\mathbb{N}, \mathcal{E})$, by Theorem $3,(\mathbb{N}, \mathcal{E})$ is $\delta$-rigid. By Theorem 2 with $Y=2 \mathbb{N},(\mathbb{N}, \mathcal{E})$ is not $\lambda$-rigid.

3. Ordinal and submetrizable balleans. We recall that a ballean $(X, \mathcal{E})$ is ordinal if $\mathcal{E}$ has a base linearly ordered by inclusion. In this case $\mathcal{E}$ has a base well-ordered by inclusion.

A ballean $X, \mathcal{E}$ is called metrizable if there exists a metric $d$ on $X$ such that $\mathcal{E}$ has the base $\left\{\{(x, y): d(x, y)<r\}: r \in \mathbb{R}^{+}\right\}$. A ballean $(X, \mathcal{E})$ is metrizable if and only if $\mathcal{E}$ has a countable base [12, Theorem 2.1.1]. Hence, every metrizable ballean is ordinal.

We denote by $\mathcal{M}$ and $\mathcal{L}$ the cases of metrizable and ordinal balleans.

Theorem 4. The following statements hold:

1. every ordinal ballean is $\lambda$-strong;

2. $\mathcal{M}$ and $\mathcal{L}$ are $\lambda$-rigid;

3. an ordinal ballean $(X, \mathcal{E})$ is $\lambda$-rigid if and only if $(X, \mathcal{E})$ is discrete;

4. $\mathcal{M}$ and $\mathcal{L}$ are not $\lambda$-stable.

Proof. (1) let $(X, \mathcal{E})$ be an unbounded ordinal ballean, $\left\{L_{\lambda}: \lambda<\kappa\right\}$ be a base of $\mathcal{E}$ wellordered by inclusion. We take a ballean $\left(X, \mathcal{E}^{\prime}\right) \lambda$-equivalent to $(X, \mathcal{E})$ and show that $\mathcal{E}^{\prime} \subseteq \mathcal{E}$. Assume the contrary and choose $E^{\prime} \in \mathcal{E}^{\prime} \backslash \mathcal{E}$. Then we can choose a $\kappa$-sequence $\left(x_{\alpha}\right)_{\alpha<\kappa}$ in $X$ such that

- $L_{\alpha}\left[x_{\alpha}\right] \cap L_{\beta}\left[x_{\beta}\right]=\varnothing, \alpha<\beta<\kappa ;$

- $E^{\prime}\left[x_{\alpha}\right] \cap E^{\prime}\left[x_{\beta}\right]=\varnothing, \alpha<\beta<\kappa$;

- $E^{\prime}\left[x_{\alpha}\right] \backslash L_{\alpha}\left[x_{\alpha}\right] \neq \varnothing, \alpha<\kappa$.

For each $\alpha<\kappa$, we pick $y_{\alpha} \in E^{\prime}\left[x_{\alpha}\right] \backslash L_{\alpha}\left[x_{\alpha}\right]$ and put $X^{\prime}=\left\{x_{\alpha}: \alpha<\kappa\right\}, Y=\left\{y_{\alpha}: \alpha<\kappa\right\}$. Then $X^{\prime}, Y$ are close in $\left(X, \mathcal{E}^{\prime}\right)$. For each $E \in \mathcal{E}$, there exists $\beta<\kappa$ such that $E\left[x_{\alpha}\right] \subseteq L_{\alpha}\left[x_{\alpha}\right]$, $\alpha<\beta$. Hence, $E\left[X^{\prime}\right] \cap Y$ is bounded and $X^{\prime}, Y$ are not linked in $\mathcal{E}$ contradicting $\lambda$-equivalence of $(X, \mathcal{E})$ and $\left(X, \mathcal{E}^{\prime}\right)$.

(2) follows from (1).

(3) We note that every unbounded subset of an ordinal ballean can be partitioned in two unbounded subsets and apply Theorem 2 .

(4) follows from Theorem 2.

By Theorem 3, the statement (3) of Theorem 4 does not hold for $\delta$ in place of $\lambda$.

Question 1. Is every metrizable ballean $\delta$-rigid? Equivalently, is $\mathcal{M} \delta$-stable? 
We recall that an unbounded ballean $(X, \mathcal{E})$ is submetrizable if there exists a coarse structure $\mathcal{E}^{\prime}$ on $X$ such that $\mathcal{E} \subseteq \mathcal{E}^{\prime}$ and $\left(X, \mathcal{E}^{\prime}\right)$ is unbounded and metrizable.

For a ballean $(X, \mathcal{E})$, a function $f: X \longrightarrow \mathbb{R}$ is called macro-uniform if, for every $E \in \mathcal{E}$, there exists $r_{E} \in \mathbb{R}^{+}$such that $\operatorname{diam} f(E[x]) \leq r_{E}$ for each $x \in X$. We denote $\operatorname{mu}(X, \mathcal{E})$ the family of all macro-uniform functions on $X$.

By [13, Theorem 2.2.3], an unbounded ballean $(X, \mathcal{E})$ is submetrizable if and only if there exists an unbounded function $f \in \mathrm{mu}(X, \mathcal{E})$.

Theorem 5. If balleans $(X, \mathcal{E}),\left(X, \mathcal{E}^{\prime}\right)$ are $\lambda$-equivalent, then $\mathrm{mu}(X, \mathcal{E})=\mathrm{mu}\left(X, \mathcal{E}^{\prime}\right)$.

Proof. We assume that there exists $f \in \operatorname{mu}\left(X, \mathcal{E}^{\prime}\right) \backslash \mathrm{mu}(X, \mathcal{E})$ and choose $E \in \mathcal{E}$ and a sequence $\left(y_{n}\right)_{n \in \omega}$ in $X$ such that diam $f\left(E\left[y_{n}\right]\right)>2 n$. For each $n \in \omega$, we pick $z_{n} \in E\left[y_{n}\right]$ such that $\left|f\left(y_{n}\right)\right|-f\left(z_{n}\right) \mid>n$. Passing to subsequences, we may suppose that $\left|f\left(y_{n}\right)\right|-f\left(z_{m}\right) \mid>n$ for all $m \geq n$. We denote $Y=\left\{y_{n}: n \in \omega\right\}, Z=\left\{z_{n}: n \in \omega\right\}$ and note that $Y \delta_{(X, \mathcal{E})} Z$. Since $f \in \mathrm{mu}\left(X, \mathcal{E}^{\prime}\right)$, for every $E^{\prime} \in \mathcal{E}^{\prime}$, there exists $k \in \mathbb{N}$ such that $\operatorname{diam} f\left(\mathcal{E}^{\prime}\left[y_{n}\right]\right)<k$ for all $n \in \omega$. Then $\mathcal{E}^{\prime}[Y] \cap Z$ is finite, so $Y, Z$ are not linked in $\left(X, \mathcal{E}^{\prime}\right)$.

Corollary 2. The class of submetrizable balleans is $\lambda$-stable.

A ballean $(X, \mathcal{E})$ is called mu-bounded if $(X, \mathcal{E})$ is not submetrizable. For mu-bounded balleans, see [2]. By Corollary 2, the class of mu-bounded balleans is $\lambda$-stable.

4. Normal balleans. For a ballean $(X, \mathcal{E})$, two subsets $A, B$ of $X$ are called asymptotically disjoint if for every $E \in \mathcal{E}$ the intersection $E[A] \cap E[B]$ is bounded. We note that unbounded subsets $A, B$ are asymptotically disjoint if and only if $A, B$ are not linked.

A subset $U$ of $X$ is called an asymptotic neighbourhood of a subset $A$ if for every $E \in \mathcal{E}$ the set $E[A] \backslash U$ is bounded.

A ballean $(X, \mathcal{E})$ is called normal [7] if any two asymptotically disjoint subsets have disjoint asymptotic neighbourhoods.

A function $f: X \longrightarrow \mathbb{R}$ is called slowly oscillating if for any $E \in \mathcal{E}$ and $\varepsilon>0$ there exists a bounded subset $B$ of $X$ such that $\operatorname{diam} f(E[x])<\varepsilon$ for each $x \in X$. By [6, Theorem 2.2], a ballean $(X, \mathcal{E})$ is normal if and only if, for any two disjoint and asymptotically disjoint subsets $A, B$ of $X$, there exists a slowly oscillating function $f: X \longrightarrow[0,1]$ such that $\left.f\right|_{A}=0$, $\left.f\right|_{B}=1$.

We denote by $\operatorname{so}(X, \mathcal{E})$ and $\operatorname{sob}(X, \mathcal{E})$ the families of all and all bounded slowly oscillating functions on $X$.

Theorem 6. If balleans $(X, \mathcal{E}),\left(X, \mathcal{E}^{\prime}\right)$ are $\lambda$-equivalent then $\operatorname{sob}(X, \mathcal{E})=\operatorname{sob}\left(X, \mathcal{E}^{\prime}\right)$.

Proof. We suppose the contrary and let $f \in \operatorname{sob}\left(X, \mathcal{E}^{\prime}\right) \backslash \operatorname{sob}(X, \mathcal{E})$. We denote $\mathcal{B}=\mathcal{B}_{(X, \mathcal{E})}=$ $\mathcal{B}_{\left(X, \mathcal{E}^{\prime}\right)}$ and take $\varepsilon>0$ and $E \in \mathcal{E}$ such that, for every $B \in \mathcal{B}$, there exists $x_{B} \in X \backslash B$ such that $\operatorname{diam} f\left(E\left[x_{B}\right]\right)>\varepsilon$. We pick $y_{B} \in E\left[x_{B}\right]$ such that $\left|f\left(x_{B}\right)-f\left(y_{B}\right)\right|>\varepsilon$. Then we enumerate $\mathcal{B}=\left\{B_{\alpha}: \alpha<\kappa\right\}$, denote $Z=\left\{x_{B}: B \in \mathcal{B}\right\}$ and define a mapping $h: Z \longrightarrow X$ as follows.

We put $h\left(x_{B_{0}}\right)=y_{B_{0}}$ and let $h$ is already defined for each $x_{B_{\alpha}}, \alpha<\beta$. If $h$ is not defined at $x_{B_{\beta}}$ then we put $f\left(x_{B_{\beta}}\right)=y_{B_{\beta}}$.

We take an ultrafilter $p \in \mathcal{B}^{\sharp}$ such that $Z \in p$, denote $q=h^{\beta}(p)$ and observe that $\left|f^{\beta}(p)-f^{\beta}(q)\right| \geq \varepsilon$. We choose $P \in p, Q \in p$ such that $P \subseteq Z, Q \subseteq Z,\left|f(x)-f^{\beta}(p)\right|<\frac{1}{2} \varepsilon$, $x \in P$ and $\left|f(x)-f^{\beta}(q)\right|<\frac{1}{2} \varepsilon, x \in Q$. By the construction of $h$, we have $P \delta_{(X, \mathcal{E})} Q$, so $P \lambda_{(X, \mathcal{E})} Q$. By the assumption, $P \lambda_{\left(X, \mathcal{E}^{\prime}\right)} Q$. Hence, there exist $E^{\prime} \in \mathcal{E}^{\prime}$ and an unbounded 
$P^{\prime} \subseteq P$ such that $E^{\prime}[x] \cap Q \neq \varnothing$ for each $x \in P^{\prime}$. Since $f$ is slowly oscillating in $\left(X, \mathcal{E}^{\prime}\right)$, there exists $B \in \mathcal{B}$ such that diam $f\left(E^{\prime}[x]\right)<\frac{1}{2} \varepsilon$ for each $x \in X \backslash B$. Then $f\left(E^{\prime}[x]\right) \cap Q=\varnothing$ for each $x \in P^{\prime} \backslash B$ and we get a contradiction with the choice of $P^{\prime}$.

Corollary 3. The class of normal balleans is $\lambda$-stable.

If $(X, \mathcal{E}),\left(X, \mathcal{E}^{\prime}\right)$ are metrizable and $\operatorname{sob}(X, \mathcal{E})=\operatorname{sob}\left(X, \mathcal{E}^{\prime}\right)$, then $\lambda_{(X, \mathcal{E})}=\lambda_{\left(X, \mathcal{E}^{\prime}\right)}$ and, by Theorem $4(2), \mathcal{E}=\mathcal{E}^{\prime}$.

We show that the class of normal balleans is not $\delta$-rigid.

Example 2. We denote by $S_{\omega}$ the group of all permutations of $\omega$, by $\mathcal{E}$ the coarse structure on $\omega$ with the base

$$
\left\{\{(x, y): y \in\{x\} \cup F x\}: F \in\left[S_{\omega}\right]^{<\omega}\right\}
$$

and take the strongest coarse structure $\mathcal{E}^{\prime}$ on $\omega$ such that $\mathcal{B}_{\left(\omega, \mathcal{E}^{\prime}\right)}=[\omega]^{<\omega}$, see Example 3 in [3]. Then $\mathcal{E} \subsetneq \mathcal{E}^{\prime}$ and every infinite subset of $\omega$ is large in $(\omega, \mathcal{E})$ and $\left(\omega, \mathcal{E}^{\prime}\right)$. Hence, $\delta_{(\omega, \mathcal{E})}=\delta_{\left(\omega, \mathcal{E}^{\prime}\right)}$ and $(\omega, \mathcal{E}),\left(\omega, \mathcal{E}^{\prime}\right)$ are normal because any two infinite subsets of $X$ are not asymptotically disjoint in $(\omega, \mathcal{E})$ and $\left(\omega, \mathcal{E}^{\prime}\right)$.

Question 2. Is so $(X, \mathcal{E})=\operatorname{so}\left(X, \mathcal{E}^{\prime}\right)$ for any $\lambda$-equivalent balleans $(X, \mathcal{E}),\left(X, \mathcal{E}^{\prime}\right)$ ?

Following [3], we say that a ballean $(X, \mathcal{E})$ has bounded growth if, there exists a mapping $f: X \longrightarrow \mathcal{B}_{(X, \mathcal{E})}$ such that for every $E \in \mathcal{E}$, the set $\{x \in X: E[x] \not f f(x)\}$ is bounded.

Question 3. Is the class of balleans of bounded growth $\lambda$-stable?

5. Finitary balleans. For a group $G$, we denote by $\mathcal{F}_{G}$ the coarse structure on $G$ with the base

$$
\left\{\{(x, y) \in G \times G: y \in\{x\} \cup F x\}: F \in[G]^{<\omega}\right\}
$$

and say that $\left(G, \mathcal{F}_{G}\right)$ is the finitary group ballean of $G$. The following theorem show that the class of finitary group balleans is $\lambda$-rigid.

Theorem 7. Let $G, H$ be group on a set $X$ such that $\lambda_{\left(X, \mathcal{F}_{G}\right)}=\lambda_{\left(X, \mathcal{F}_{H}\right)}$. Then $\mathcal{F}_{G}=\mathcal{F}_{H}$.

Proof. If $X$ is countable then the statement follows from Theorem $4(2)$ because $\mathcal{F}_{G}, \mathcal{F}_{H}$ have countable bases, so we suppose that $X$ is uncountable.

We denote by $\cdot$, * the group operations in $G, H$ and, on the contrary, assume that $\mathcal{F}_{G} \backslash \mathcal{F}_{H} \neq \varnothing$. Then there exists $F \in[X]^{<\omega}$ such that, for any $H \in[X]^{<\omega}$, there exists $x \in X$ such that $F \cdot x \backslash H * x \neq \varnothing$.

We use the following observation:

( $\star$ ) for every countable subset $Y$ of $X$, there exists a countable subset $Z$ such that $Y \subset Z$ and, for every $A \in[Y]^{<\omega}$, there exists $z \in Z$ such that $F \cdot z \backslash A * z \neq \varnothing$.

We fix an arbitrary countable subset $Y_{0}$ of $X$ containing $F$ and apply $(\star)$ to get a countable subgroup $Z_{0}$ of $G, Y_{0} \subset Z_{0}$. Then we denote by $Y_{1}$ the subgroup of $H$ generated by $Z_{0}$. Analogously, for $Y_{1}$ we use $(\star)$ to get the subgroup $Z_{1}$ of $G, Y_{1} \subset Z_{1}$, and denote by $Y_{2}$ the subgroup of $H$ generated by $Z_{1}$. After $\omega$ steps, we put $S=\bigcup_{n \in \omega} Y_{n}$ and note that $S$ is a subgroup of $G$ and $S$ is a subgroup of $H$.

By the construction of $S$, for every $A \in[S]^{<\omega}$, there exists $x \in S$ such that $F \cdot x \backslash A * x \neq \varnothing$. Hence, the restriction of $\mathcal{F}_{G}$ and $\mathcal{F}_{H}$ to $S$ are distinct. Since $S$ is countable, we get a contradiction to Theorem 4(2). 
A ballean $(X, \mathcal{E})$ is called finitary if for every $E \in \mathcal{E}$ there exists $n \in \omega$ such that $|E[x]|<n$ for each $x \in X$. By [9], for every finitary ballean $(X, \mathcal{E})$, there exists a group $S$ of permutations of $X$ such that $\mathcal{E}$ has the base

$$
\left\{\{(x, y): y \in\{x\} \cup F x\}: F \in[S]^{<\omega}\right\} .
$$

Example 2 shows that the class of finitary balleans is not $\delta$-stable.

Question 4. Is the class of finitary balleans $\delta$-rigid?

The product of two $\lambda$-rigid balleans needs not to be $\lambda$-rigid: take two metrizable unbounded discrete balleans and apply Corollary 1 and Theorem 2 .

Question 5. Is the product of two $\delta$-rigid balleans $\delta$-rigid?

A ballean $(X, \mathcal{E})$ is called cellular if $\mathcal{E}$ has a base consisting of equivalence relations. Equivalently [4, Theorem 3.1.3], $(X, \mathcal{E})$ is cellular if $\operatorname{asdim}(X, \mathcal{E})=0$.

Question 6. Is the class of cellular balleans $\lambda$-stable?

Remark 1. As was recently shown by Banakh [1], the answers to Questions 4 and 6 are negative under $\mathrm{CH}$ (more precisely, under $\mathfrak{b}=\mathfrak{c}$, and $\Delta_{\omega}^{\circ}=\mathfrak{c}$, respectively).

5. Ultrafilters. Let $(X, \mathcal{E})$ be a ballean. We endow $X$ with the discrete topology and consider the Stone-Cech compactification $\beta X$ of $X$. We take the points of $\beta X$ to be the ultrafilters on $X$. Then $\mathcal{B}_{(X, \mathcal{E})}^{\sharp}$ is a closed subset of $\beta X$.

Given any $r, q \in \mathcal{B}_{(X, \mathcal{E})}^{\sharp}$, we say that $r, q$ are parallel (and write $r \|_{(X, \mathcal{E})} q$ ) if there exists $E \in \mathcal{E}$ such that $E[P] \in q$ for each $P \in p$. By [7, Lemma 4.1], $\|_{(X, \mathcal{E})}$ is an equivalence on $\mathcal{B}_{(X, \mathcal{E})}^{\sharp}$. We denote by $\sim_{(X, \mathcal{E})}$ the minimal (by inclusion) closed (in $\mathcal{B}_{(X, \mathcal{E})}^{\sharp} \times \mathcal{B}_{(X, \mathcal{E})}^{\sharp}$ ) equivalence such that $\|_{(X, \mathcal{E})} \subseteq \sim_{(X, \mathcal{E})}$. The quotient $\nu(X, \mathcal{E})$ is called the Higson corona of $(X, \mathcal{E})$.

By [8, Proposition 1], $r \sim_{(X, \mathcal{E})} q$ if and only if $h^{\beta}(p)=p^{\beta}(q)$ for every slowly oscillating function $h: X \longrightarrow[0,1]$.

Theorem 8. Let $\mathcal{E}, \mathcal{E}^{\prime}$ be coarse structures on a set $X$ such that $\mathcal{B}_{(X, \mathcal{E})}^{\sharp}=\mathcal{B}_{\left(X, \mathcal{E}^{\prime}\right)}^{\sharp}$ and $\left\|_{(X, \mathcal{E})}=\right\|_{\left(X, \mathcal{E}^{\prime}\right)}$. Then $(X, \mathcal{E}),\left(X, \mathcal{E}^{\prime}\right)$ are $\delta$-equivalent. If $\mathcal{B}_{(X, \mathcal{E})}^{\sharp}$ is finite, then $\mathcal{E}=\mathcal{E}^{\prime}$.

Proof. We suppose that there exist $P \subseteq X, Q \subseteq X$ such that $P \delta_{(X, \mathcal{E})} Q$ and $P \backslash E^{\prime}(Q) \neq \varnothing$ for each $E^{\prime} \in \mathcal{E}^{\prime}$.

Since $P \delta_{(X, \mathcal{E})} Q$, there exists $E \in \mathcal{E}$ such that $P \subseteq E[Q]$ and $E=E^{-1}$. For each $x \in P$, we pick $f(x) \in Q$ such that $(x, f(x)) \in E$. If $p \in \mathcal{B}_{(X, \mathcal{E})}^{\sharp}$ and $P \in p$ then $p \|_{(X, \mathcal{E})} f^{\beta}(p)$ and $Q \in f^{\beta}(p)$. Thus, each ultrafilter $p \in \mathcal{B}_{(X, \mathcal{E})}^{\sharp}$ such that $P \in p$ is parallel in $\mathcal{B}_{(X, \mathcal{E})}^{\sharp}$ to some ultrafilter $q$ such that $Q \in q$.

We take an arbitrary ultrafilter $r$ such that $P \backslash E^{\prime}[Q] \in r$ for each $E^{\prime} \in \mathcal{E}$. Then $P \in r$ and $r$ is not parallel in $\mathcal{B}_{\left(X, \mathcal{E}^{\prime}\right)}^{\sharp}$ to each ultrafilter $q$ such that $Q \in q$. Hence, $\left\|_{(X, \mathcal{E})} \neq\right\|_{\left(X, \mathcal{E}^{\prime}\right)}$.

To prove the second statement, it suffices to show that $(X, \mathcal{E})$ is coarsely discrete and apply Theorem 3 .

We choose a representative from each class of parallel ultrafilter in $\mathcal{B}_{(X, \mathcal{E})}^{\sharp}$. Let $p_{1}, \ldots, p_{m}$ be the set of obtained representatives. Let $E \in \mathcal{E}$. We choose $P_{1} \in p_{1}, \ldots, P_{n} \in p_{n}$ such that $E\left[P_{i}\right] \cap P_{j}=\varnothing$. Then $P_{1} \cup, \ldots, P_{n}$ is discrete and large in $(X, \mathcal{E})$, so $(X, \mathcal{E})$ is coarsely discrete. 
Remark 2. Let $\mathcal{B}_{(X, \mathcal{E})}^{\sharp}=\mathcal{B}_{\left(X, \mathcal{E}^{\prime}\right)}^{\sharp}$. Does $\delta_{(X, \mathcal{E})}=\delta_{\left(X, \mathcal{E}^{\prime}\right)}$ implies $\left\|_{(X, \mathcal{E})}=\right\|_{\left(X, \mathcal{E}^{\prime}\right)}$ ?

The answer to this question is negative. We take the balleans $(\omega, \mathcal{E}),\left(\omega, \mathcal{E}^{\prime}\right)$ from Example 11 and note that $p, q \in \omega^{*}$ are parallel in $\mathcal{B}_{(\omega, \mathcal{E})}^{\sharp}$ if and only if $p=f^{\beta}(q)$ for some bijection $f: \omega \longrightarrow \omega$. On the other hand, we partition $\omega=\bigcup_{n \in \omega} W_{n}$ so that $\left|W_{n}\right|=n+1$ and take a mapping $h: \omega \longrightarrow \omega$ such that $h\left(W_{n}\right)=\left\{a_{n}\right\}, a_{n} \in W_{n}$. We choose $p \in \omega^{*}$ such that $p$ and $h^{\beta}(p)$ are not isomorphic. By the choice of $h, p$ and $h^{\beta}(p)$ are parallel in $\mathcal{B}_{\left(X, \mathcal{E}^{\prime}\right)}^{\sharp}$ but not in $\mathcal{B}_{(X, \mathcal{E})}^{\sharp}$.

Theorem 9. If $(X, \mathcal{E}),\left(X, \mathcal{E}^{\prime}\right)$ are finitary balleans and $\left\|_{(X, \mathcal{E})}=\right\|_{\left(X, \mathcal{E}^{\prime}\right)}$ then $\mathcal{E}=\mathcal{E}^{\prime}$.

Proof. Let $G, G^{\prime}$ be groups of permutations of $X$ defining $\mathcal{E}, \mathcal{E}^{\prime}$. We assume that $\mathcal{E} \backslash \mathcal{E}^{\prime} \neq \varnothing$. Then there is $H \in[G]^{<\omega}$ such that, for every $A \in\left[G^{\prime}\right]^{<\omega}$, the set $Y_{A}=\{x \in X: H x \backslash A x \neq \varnothing\}$ is infinite. We take $p \in \mathcal{B}_{(X, \mathcal{E})}^{\sharp}$ such that $Y_{A} \in p$ for each $A \in\left[G^{\prime}\right]^{<\omega}$. Let $H=\left\{h_{1}, \ldots, h_{n}\right\}$. We take $g_{1}, \ldots, g_{n} \in G^{\prime}$ such that $h_{1} p=g_{1} p, \ldots, h_{n} p=g_{n} p$ and put $B=\left\{g_{1}, \ldots, g_{n}\right\}$. Then $\left\{x \in X: h_{1} x=g_{1} x, \ldots, h_{n} x=g_{n} x\right\} \in p$ and $Y_{B} \notin p$, contradict the choice of $p$.

Theorem 10. For a normal ballean $(X, \mathcal{E})$ and $p, q \in \mathcal{B}_{(X, \mathcal{E})}^{\sharp}$, the following statements are equivalent:

(1) $p \sim q$;

(2) $P \lambda Q$ for each $P \in p, Q \in q$;

(3) $(p, q) \in c l \|$.

Proof. (1) $\Rightarrow(2)$. If $P, Q$ are unbounded and asymptotically disjoint then, by the normality of $(X, \mathcal{E})$, there exists a slowly oscillating function $f: X \longrightarrow[0,1]$ such that $\left.f\right|_{P}=1,\left.f\right|_{Q}=0$, so $f^{\beta}(p) \neq f^{\beta}(q)$ and $p, q$ are not equivalent.

$(2) \Rightarrow(3)$. We choose $E \in \mathcal{E}$, unbounded subsets $P^{\prime} \subseteq P, Q^{\prime} \subseteq Q$ such that there is a bijection $h: P^{\prime} \longrightarrow Q^{\prime}$ satisfying $(x, h(x)) \in E, x \in P^{\prime}$. If $r \in \mathcal{B}_{(X, \mathcal{E})}^{\sharp}$ and $P^{\prime} \in r$ then $r \| h^{\beta}(r)$ and $P \in r, Q \in h^{\beta}(r)$.

The implication $(3) \Rightarrow(1)$ follows from definitions of $\sim$ and $\|$.

Theorem 11. A ballean $(X, \mathcal{E})$ is normal if and only if $\sim=c l \|$.

Proof. By Theorem 10, if $(X, \mathcal{E})$ is normal then $\sim=c l \|$. We assume that $\sim=c l \|$ and show that any two unbounded asymptotically disjoint subsets $P, Q$ of $X$ have disjoint asymptotic neighborhoods.

Let $p, q \in \mathcal{B}_{(X, \mathcal{E})}^{\sharp}, P \in p, Q \in q$. Since $P, Q$ are asymptotically disjoint, we have $(p, q) \notin$ $c l \|$, so there exists a slowly oscillating function $f: X \longrightarrow[0,1]$ such that $f^{\beta}(p)=1, f^{\beta}(q)=$ 0 . We denote $P^{\prime}=\{x \in P: f(x)>3 / 4\}, Q^{\prime}=\{x \in Q: f(x)<1 / 2\}$. Then $P^{\prime} \in p, Q^{\prime} \in q$ and $P^{\prime}, Q^{\prime}$ have disjoint asymptotic neighborhoods.

We fix $p \in \mathcal{B}_{(X, \mathcal{E})}^{\sharp}$ such that $P \in p$ and apply above paragraph to find $q_{1}, \ldots, q_{n} \in \mathcal{B}_{(X, \mathcal{E})}^{\sharp}$, $Q_{1} \in q_{1}, \ldots, Q_{n} \in q_{n}$ and $P^{\prime} \in p$ such that $P^{\prime}$ and $Q_{1} \cup \cdots \cup Q_{n}$ have asymptotically disjoint neighborhoods and $Q \backslash\left(Q_{1} \cup \cdots \cup Q_{n}\right)$ is bounded. It follows that $P^{\prime}, Q$ have disjoint asymptotic neighborhoods.

At last, we find $p_{1}, \ldots, p_{m} \in \mathcal{B}_{(X, \mathcal{E})}^{\sharp}$ and $P_{1} \in p_{1}, \ldots, P_{m} \in p_{m}$ such that $p_{1} \cup \cdots \cup p_{m}$ and $Q$ have disjoint asymptotic neighborhoods and $P \backslash\left(P_{1} \cup \cdots \cup P_{m}\right)$ is bounded. Hence, $P$ and $Q$ have disjoint asymptotic neighborhoods. 


\section{REFERENCES}

1. T. Banakh, Small uncountable cardinals in large-scale topology, preprint arxiv.org/abs/2002.08800.

2. T. Banakh, I. Protasov, Functional boundedness of balleans: coarse versions of compactness, Axioms, 2019, 8, 33; doi: 10.3390/axioms 8010033.

3. T. Banakh, I. Protasov, The normality and bounded growth of balleans, arXiv: 1810.07979.

4. T. Banakh, I. Protasov, Minmax bornologies, Ukr. Math. Bull., 16 (2019), 496-502.

5. D. Dikranjan, I. Protasov, K. Protasova, N. Zava, Balleans, hyperballeans and ideals, Appl. Gen. Topology, 20 (2019), 431-447.

6. O. Petrenko, I. Protasov, Balleans and filters, Math. Stud., 38 (2012), 3-11.

7. I. Protasov, Normal ball structures, Math. Stud., 20 (2003), 3-16.

8. I. Protasov, Coronas of balleans, Topology Applications, 149 (2005), 149-160.

9. I. Protasov, Asymptotic proximities, Appl. Gen. Topology, 9 (2008), 189-195.

10. I.V. Protasov, Balleans of bounded geometry and G-space, Algebra Discrete Math., 2008, №2, $101-108$.

11. I. Protasov, T. Banakh, Ball structures and colorings of groups and graphs, Math. Stud. Monogr. Ser., V.11, VNTL, Lviv, 2003.

12. I. Protasov, K. Protasova, On hyperballeans of bounded geometry, Europ. J. Math., 4 (2018), 1515-1520. 13. I. Protasov, M. Zarichnyi, General Asymptopogy, Math. Stud. Monogr., V.12, VNTL, Lviv, 2007.

14. J. Roe, Lectures on Coarse Geometry, AMS University Lecture Ser., V.31, Providence, RI, 2003.

Faculty of Computer Science and Cybernetics

Kyiv University, Kyiv, Ukraine

i.v.protasov@gmail.com

ksuha@freenet.com.ua 